7-1-2011

\title{
On active disturbance rejection based control design for superconducting RF cavities
}

John Vincent

Michigan State University

Dan Morris

Michigan State University Foflow this and additional works at: https://engagedscholarship.csuohio.edu/enece_facpub

地视 błsther Electrical and Computer Engineering Commons Michigan State University.

How does access to this work benefit you? Let us know!

\section{Ëlidphissterés Statement}

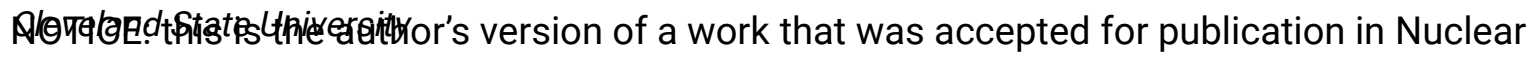
Shstrumentys and Methods in Physics Research Section A: Accelerators, Spectrometers,

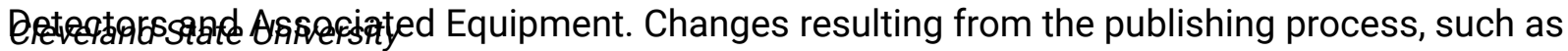
peer review, editing, corrections, structural formatting, and other quality control mechanisms may not be reflected in this document. Changes may have been made to this work since it was Submitted por for additional authors Instruments and Methods in Physics Research Section A: Accelerators, Spectrometers, Detectors and Associated Equipment, 643, 1, (07-01-2011); 10.1016/j.nima.2011.04.033

\section{Repository Citation}

Vincent, John; Morris, Dan; Usher, Nathan; Gao, Zhiqiang; Zhaob, Shen; Nicoletti, Achille; and Zheng, Qinling, "On active disturbance rejection based control design for superconducting RF cavities" (2011). Electrical Engineering \& Computer Science Faculty Publications. 260.

https://engagedscholarship.csuohio.edu/enece_facpub/260

This Article is brought to you for free and open access by the Electrical Engineering \& Computer Science Department at EngagedScholarship@CSU. It has been accepted for inclusion in Electrical Engineering \& Computer Science Faculty Publications by an authorized administrator of EngagedScholarship@CSU. For more information, please contact library.es@csuohio.edu. 


\section{Authors}

John Vincent, Dan Morris, Nathan Usher, Zhiqiang Gao, Shen Zhaob, Achille Nicoletti, and Qinling Zheng

This article is available at EngagedScholarship@CSU: https://engagedscholarship.csuohio.edu/enece_facpub/260 


\title{
Active Disturbance Rejection Control for MEMS Gyroscopes
}

\author{
Qing Zheng, Lili Dong, Dae Hui Lee, and Zhiqiang Gao
}

\begin{abstract}
A new control method is presented to drive the drive axis of a Micro-Electro-Mechanical Systems (MEMS) gyroscope to resonance and to regulate the output amplitude of the axis to a fixed level. It is based on a unique active disturbance rejection control (ADRC) strategy, which actively estimates and compensates for internal dynamic changes of the drive axis and external disturbances in real time. The stability analysis shows that both the estimation error and the tracking error of the drive axis output are bounded and that the upper bounds of the errors monotonously decrease with the increase of the controller bandwidth. The control system is simulated and tested using a field-programmable-gatearray-based digital implementation on a piezoelectric vibrational gyroscope. Both simulation and experimental results demonstrate that the proposed controller not only drives the drive axis to vibrate along the desired trajectory but also compensates for manufacture imperfections in a robust fashion that makes the performance of the gyroscope insensitive to parameter variations and noises. Such robustness, the fact that the control design does not require an accurate plant model, and the ease of implementation make the proposed solution practical and economic for industrial applications.
\end{abstract}

Index Terms-Active disturbance rejection control (ADRC), discrete implementation, extended state observer (ESO), field-programmable gate array (FPGA), Micro-Electro-Mechanical Systems (MEMS) gyroscopes.

\section{INTRODUCTION}

M ICRO-ELECTRO-MECHANICAL Systems (MEMS) gyroscope is a micro- or millimeter-scale inertial rate sensor. It has been used in automobiles (stability control and GPS), aerospace (GPS-assisted inertial navigation), and consumer electronics (camera image stabilization and 3-D mouse) [1]. Compared to electromechanical gyroscopes, the MEMS gyroscope is small in size, inexpensive, and energy efficient. A control system is generally used to excite the vibration along two vibrating modes (driving and sensing modes) of the MEMS gyroscope and to estimate the rotation rate. However, the small size of the MEMS gyroscope puts a big challenge on controller design and microfabrication. The imprecise microfabrication and disturbances result in mechanical coupling terms between two axes, mechanical-thermal noises, and parameter variations, which consequently degrade the performance of the MEMS gyroscope. Therefore, a closed-loop control system is essential for improving the performance of the MEMS gyroscope through effectively compensating for the mechanical imperfections and the disturbances in control efforts.

Since the 1990s, there has been a limited amount of research on feedback control system designs for MEMS gyroscopes. The controllers introduced in [2]-[4] did not fully account for the mechanical coupling terms on the drive axis caused by the manufacture imperfections. The adaptive controllers in [5] and [6] achieved performance improvement in a noise-free setting. The adaptive controller in [7] is designed for the MEMS gyroscopes operating in an adaptive mode. However, most reported MEMS gyroscopes operate in the conventional mode [8] where the movement of the mass along the drive axis is relatively large and the movement along the sense axis is very small. The controller in [8] regulates the vibration along the sensing mode of the MEMS gyroscope, while the control of the driving mode is disregarded.

In this paper, a practical solution based on the active disturbance rejection control (ADRC) technology is applied to the driving mode of the conventional MEMS gyroscope [9], [10]. The ADRC has been successfully employed in many mechanical systems [11]-[14]. However, the employment of the ADRC onto MEMS is rather new. The basic idea of this control strategy is to estimate the plant dynamics and disturbances using an extended state observer (ESO) and to actively compensate for the disturbance in control effort. With the accurate estimation of the plant dynamics and disturbances by ESO, the ADRC can successfully drive the output of the drive axis to resonance. Since the ADRC does not depend on an accurate model of the axis, it is very robust against parameter variations, disturbances, and noise. Another advantage of the controller is its few tuning parameters, making the controller easy to implement in the real world. To test the effectiveness of the ADRC, a field-programmable-gate-array (FPGA)-based digital implementation of the controller is conducted on a piezoelectrically driven vibrational beam gyroscope. The experimental results demonstrate the effectiveness of the controller.

This paper is organized as follows. The dynamics of MEMS gyroscopes is described in Section II. The ADRC approach and its stability analysis are presented in Section III. Software simulation and hardware test results are shown in Section IV. This paper ends with concluding remarks in Section V.

\section{DYNAMICS OF MEMS GYROSCOPES}

The mechanical structure of the MEMS gyroscope can be understood as a proof mass attached to a rigid frame by springs and dampers, as shown in Fig. 1. As the mass is driven to resonance along the drive $(X)$ axis and the rigid frame is rotating along the rotation axis, a Coriolis acceleration will be produced along the sense $(Y)$ axis, which is perpendicular to both drive and rotation axes. The Coriolis acceleration is proportional to the amplitude of the output of the drive axis and the unknown rotation rate [1]. Therefore, we can estimate the rotation rate through sensing the 


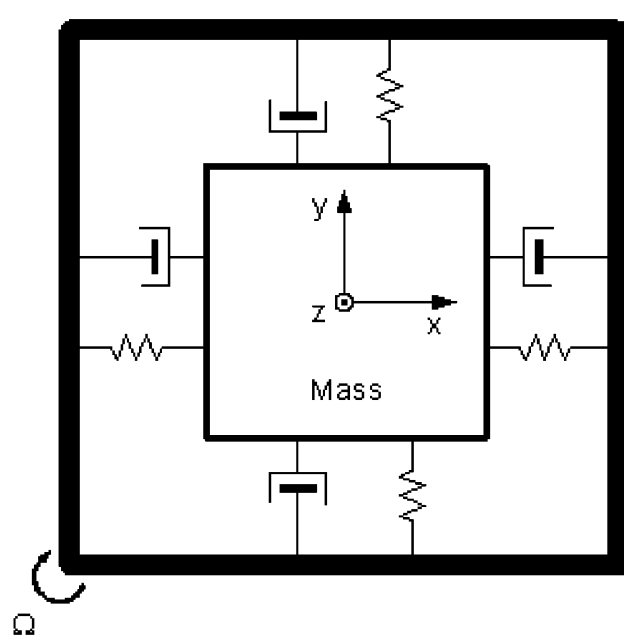

Fig. 1. Mass-spring-damper struture of MEMS gyroscopes.

vibration of the sense axis. In order to accurately sense the rotation rate, the vibrational magnitude of the drive axis has to be regulated to a fixed level. Therefore, the controller of the drive axis is mainly used to drive the drive axis to resonance and to regulate the output amplitude at a desired value.

Disregarding the damping coupling terms between two axes and assuming that the natural frequencies of both axes are the same, the vibrational MEMS gyroscope is modeled as

$$
\begin{aligned}
& \ddot{x}+2 \zeta \omega_{n} \dot{x}+\omega_{n}^{2} x+\omega_{x y} y-2 \Omega \dot{y}=\frac{k}{m} u_{d} \\
& \ddot{y}+2 \zeta \omega_{n} \dot{y}+\omega_{n}^{2} y+\omega_{x y} x+2 \Omega \dot{x}=\frac{1}{m} N(t)
\end{aligned}
$$

where $x$ and $y$ are the outputs of the drive and sense axes, respectively, $2 \Omega \dot{x}$ and $2 \Omega \dot{y}$ are the Coriolis accelerations, $\Omega$ is the rotation rate, $\omega_{n}$ is the natural frequency of the drive and sense axes, $\omega_{x y} y$ and $\omega_{x y} x$ are quadrature errors caused by spring couplings between two axes, $\zeta$ is the damping coefficient, $m$ is the mass of the MEMS gyroscope, $k$ is the controller gain, and $u_{d}$ is the control input for the drive axis. In (1), the mechanical thermal noise on the sense axis is represented by the random force $N(t)$. The effects of thermal noise on the drive axis are negligible and are ignored [15]. In the MEMS gyroscopes represented by (1), the quadrature errors are unknown constant signals, the time-varying rotation rate $\Omega$ is unknown, and the damping coefficient typically has a large varying range. In this paper, we assume that the sense axis is operating under the open-loop mode. Our control objective is to force the drive axis to oscillate at specified amplitude and resonant frequency in the presence of parameter uncertainties, mechanical couplings, and mechanical-thermal noises.

\section{ADRC}

In this paper, ADRC is employed to control the MEMS gyroscopes by dealing with modeling errors and structural uncertainties. In particular, an ESO provides an estimate of the internal dynamics of the MEMS gyroscope and the external disturbances which include the output disturbances, the unknown time-varying rotation rate, and the unknown quadrature error terms arising from mechanical imperfections. With the dynamic compensation of the estimated information, the system is reduced to a double integrator. Then, a PD controller is sufficient to control it.

We can rewrite the drive axis model in (1) as

$$
\ddot{x}=f(x, \dot{x}, d)+b u_{d}
$$

where $b=k / m ; d$ refers to the external disturbance (specifically, $d=-\omega_{x y} y+2 \Omega \dot{y}$ here); $f(x, \dot{x}, d)$, or simply donated as $f$, represents both the internal dynamics and the external disturbance; and

$$
f=-2 \zeta \omega_{n} \dot{x}-\omega_{n}^{2} x-\omega_{x y} y+2 \Omega \dot{y}
$$

The basic idea of the ADRC is to obtain the estimated $f(\hat{f})$ in real time by an ESO and to actively compensate for it in the control law. The concept of the ADRC is introduced as follows.

\section{A. ESO Design}

Let $\xi_{1}=x, \xi_{2}=\dot{x}, \xi_{3}=f$, and $\xi=\left[\begin{array}{lll}\xi_{1} & \xi_{2} & \xi_{3}\end{array}\right]^{\mathrm{T}}$. Assuming that $f$ is differentiable, the state-space form of (2) is

$$
\left\{\begin{array}{l}
\dot{\xi}=A \xi+B u_{d}+E h \\
x=C \xi
\end{array}\right.
$$

where

$$
\begin{aligned}
& A=\left[\begin{array}{lll}
0 & 1 & 0 \\
0 & 0 & 1 \\
0 & 0 & 0
\end{array}\right] \quad B=\left[\begin{array}{l}
0 \\
b \\
0
\end{array}\right] \\
& C=\left[\begin{array}{lll}
1 & 0 & 0
\end{array}\right] \quad E=\left[\begin{array}{l}
0 \\
0 \\
1
\end{array}\right]
\end{aligned}
$$

with $\xi_{3}=f$ being the augmented state and $h=\dot{f}$. A continuous ESO for (4) is designed as

$$
\left\{\begin{array}{l}
\dot{\hat{\xi}}=A \hat{\xi}+B u_{d}+L(x-\hat{x}) \\
\hat{x}=C \hat{\xi}
\end{array}\right.
$$

where $L=\left[\begin{array}{lll}l_{1} & l_{2} & l_{3}\end{array}\right]^{\mathrm{T}}$ is the observer gain vector. The observer gains are chosen such that the characteristic polynomial $s^{3}+l_{1} s^{2}+l_{2} s+l_{3}$ is Hurwitz. For tuning simplicity, all the observer poles are placed at $-\omega_{0}$. It results in the characteristic polynomial of (5) to be

$$
\lambda_{o}(s)=s^{3}+l_{1} s^{2}+l_{2} s+l_{3}=\left(s+\omega_{o}\right)^{3}
$$

where $\omega_{0}$ is the observer bandwidth of the drive axis and $L=$ $\left[\begin{array}{lll}3 \omega_{o} & 3 \omega_{o}^{3} & \omega_{o}^{3}\end{array}\right]^{\mathrm{T}}$.

Generally, the larger the observer bandwidth is, the more accurate the estimation will be. However, a large observer bandwidth will increase noise sensitivity. Therefore, a proper observer bandwidth should be selected in a compromise between the tracking performance and the noise tolerance. 


\section{B. Control Algorithm}

Once the observer is designed and well tuned, its outputs will track $\xi_{1}, \xi_{2}$, and $\xi_{3}$, respectively. By canceling the effect of $f$ using $\hat{\xi}_{3}$, the ADRC actively compensates for $f$ in real time. The ADRC control law is given by

$$
u_{d}=\frac{k_{1}\left(r-\hat{\xi}_{1}\right)+k_{2}\left(\dot{r}-\hat{\xi}_{2}\right)-\hat{\xi}_{3}+\ddot{r}}{b}
$$

where $r$ is the desired trajectory of the drive axis and $k_{1}$ and $k_{2}$ are the controller gain parameters selected to make $s^{2}+k_{2} s+k_{1}$ Hurwitz. For simplicity, let $k_{1}=\omega_{c}^{2}$ and $k_{2}=2 \omega_{c}$, where $\omega_{c}$ is the controller bandwidth. The closed-loop system for the drive axis becomes

$$
\ddot{x}=\left(f-\hat{\xi}_{3}\right)+k_{1}\left(r-\hat{\xi}_{1}\right)+k_{2}\left(\dot{r}-\hat{\xi}_{2}\right)+\ddot{r} .
$$

Note that, with a well-designed ESO, the first term on the righthand side (RHS) of (8) is negligible, and the rest of the terms on the RHS of (8) constitute a PD controller with a feedforward gain.

In practice, the controller bandwidth $\omega_{c}$ is tuned based on how fast and steady we want the output to track the set point. A large controller bandwidth generally increases the response speed, but it may push the system to its limit, leading to oscillations or even instability. Thus, the controller bandwidth should be adjusted based on the competing requirements of performance and stability margin, together with noise sensitivity. In addition, a large controller bandwidth usually increases the magnitude and rate of change in control signal and, therefore, the operation cost. The observer is tuned in a similar way: adjusting its bandwidth $\omega_{o}$ for a tradeoff between tracking performance and noise sensitivity.

The primary reason for this particular parameterization and tuning method is practicality. The observer and feedback gains must be easily tunable by most engineers, who are usually familiar with the concept and implications of bandwidth. It is advantageous that engineers could use a completely new design method without losing the critical insight gained from classical control: frequency response.

The convergence for the estimation error of ESO and the closed-loop tracking error of the ADRC is shown hereinafter.

\section{Stability}

1) Convergence of the ESO: Let $\tilde{\xi}_{i}(t)=\xi_{i}(t)-\hat{\xi}_{i}(t), i=$ $1,2,3$. From (4) and (5), the observer estimation error dynamics can be shown as
Now, let us scale the observer estimation error $\tilde{\xi}_{i}(t)$ by $\omega_{o}^{i-1}$, i.e., let $\varepsilon_{i}(t)=\left(\tilde{\xi}_{i}(t)\right) /\left(\omega_{o}^{i-1}\right), i=1,2,3$. Then, (9) can be rewritten as

$$
\dot{\varepsilon}=\omega_{o} A_{\varepsilon} \varepsilon+B_{\varepsilon} \frac{h(\xi, d)}{\omega_{o}^{2}}
$$

where

$$
A_{\varepsilon}=\left[\begin{array}{lll}
-3 & 1 & 0 \\
-3 & 0 & 1 \\
-1 & 0 & 0
\end{array}\right] \quad B_{\varepsilon}=\left[\begin{array}{l}
0 \\
0 \\
1
\end{array}\right]
$$

Theorem 1: Assuming that $h(\xi, d)$ is bounded, then there exist a constant $\sigma_{i}>0$ and a finite time $T_{1}>0$ such that $\left|\tilde{\xi}_{i}(t)\right| \leq \sigma_{i}, i=1,2,3, \forall t \geq T_{1}>0$ and $\omega_{o}>0$. Furthermore, $\sigma_{i}=\mathrm{O}\left((1) /\left(\omega_{o}^{k}\right)\right)$ for some positive integer $k$.

Proof: Solving (10), we can obtain

$$
\varepsilon(t)=e^{\omega_{o} A_{\varepsilon} t} \varepsilon(0)+\int_{0}^{t} e^{\omega_{o} A_{\varepsilon}(t-\tau)} B_{\varepsilon} \frac{h(\xi(\tau), d)}{\omega_{o}^{2}} \mathrm{~d} \tau .
$$

Let

$$
p(t)=\int_{0}^{t} e^{\omega_{o} A_{\varepsilon}(t-\tau)} B_{\varepsilon} \frac{h(\xi(\tau), d)}{\omega_{o}^{2}} \mathrm{~d} \tau .
$$

Since $h(\xi(\tau), d)$ is bounded, i.e., $|h(\xi(\tau), d)| \leq \delta$, where $\delta$ is a positive constant, it follows that

$$
\left|p_{i}(t)\right| \leq \frac{\delta}{\omega_{o}^{3}}\left[\left|\left(A_{\varepsilon}^{-1} B_{\varepsilon}\right)_{i}\right|+\left|\left(A_{\varepsilon}^{-1} e^{\omega_{o} A_{\varepsilon} t} B_{\varepsilon}\right)_{i}\right|\right]
$$

for $i=1,2,3$. Since

$$
A_{\varepsilon}^{-1}=\left[\begin{array}{lll}
0 & 0 & -1 \\
1 & 0 & -3 \\
0 & 1 & -3
\end{array}\right]
$$

one has

$$
\left|\left(A_{\varepsilon}^{-1} B\right)_{i}\right|=\left\{\begin{array}{l}
\left.1\right|_{i=1} \\
\left.3\right|_{i=2,3}
\end{array}\right.
$$

Since $A_{\varepsilon}$ is Hurwitz, there exists a finite time $T_{1}>0$ such that

$$
\left|\left[e^{\omega_{o} A_{\varepsilon} t}\right]_{i j}\right| \leq \frac{1}{\omega_{o}^{3}}
$$

for all $t \geq T_{1}, i, j=1,2,3$. Hence

$$
\left|\left[e^{\omega_{o} A_{\varepsilon} t} B\right]_{i}\right| \leq \frac{1}{\omega_{o}^{3}}
$$

for all $t \geq T_{1}, i=1,2,3$. Note that $T_{1}$ depends on $\omega_{o} A_{\varepsilon}$. Let

$$
\begin{aligned}
A_{\varepsilon}^{-1} & =\left[\begin{array}{lll}
s_{11} & s_{12} & s_{13} \\
s_{21} & s_{22} & s_{23} \\
s_{31} & s_{32} & s_{33}
\end{array}\right] \\
e^{\omega_{o} A_{\varepsilon} t} & =\left[\begin{array}{lll}
d_{11} & d_{12} & d_{13} \\
d_{21} & d_{22} & d_{23} \\
d_{31} & d_{32} & d_{33}
\end{array}\right] .
\end{aligned}
$$

$$
\begin{aligned}
& \dot{\tilde{\xi}}_{1}=\tilde{\xi}_{2}-l_{1} \tilde{\xi}_{1} \\
& \dot{\tilde{\xi}}_{2}=\tilde{\xi}_{3}-l_{2} \tilde{\xi}_{1} \\
& \dot{\tilde{\xi}}_{3}=h-l_{3} \tilde{\xi}_{1} .
\end{aligned}
$$


One has

$$
\begin{aligned}
\left|\left(A_{\varepsilon}^{-1} e^{\omega_{o} A_{\varepsilon} t} B_{\varepsilon}\right)_{i}\right|=\mid s_{i 1} d_{13}+s_{i 2} d_{23} & +s_{i 3} d_{33} \mid \\
& \leq\left\{\begin{array}{c}
\frac{1}{\omega_{o}^{3}} \\
\left.\frac{4}{\omega_{o}^{3}}\right|_{i=1,3}
\end{array}\right.
\end{aligned}
$$

for all $t \geq T_{1}$. From (13), (14), and (17), we obtain

$$
\left|p_{i}(t)\right| \leq \frac{3 \delta}{\omega_{o}^{3}}+\frac{4 \delta}{\omega_{o}^{6}}
$$

for all $t \geq T_{1}, i=1,2,3$. Let $\varepsilon_{\text {sum }}(0)=\left|\varepsilon_{1}(0)\right|+\left|\varepsilon_{2}(0)\right|+$ $\left|\varepsilon_{3}(0)\right|$. It follows that

$$
\left|\left[e^{\omega_{o} A_{\varepsilon} t} \varepsilon(0)\right]_{i}\right| \leq \frac{\varepsilon_{\mathrm{sum}}(0)}{\omega_{o}^{3}}
$$

for all $t \geq T_{1}, i=1,2,3$. From (11), one has

$$
\left|\varepsilon_{i}(t)\right| \leq\left|\left[e^{\omega_{o} A_{\varepsilon} t} \varepsilon(0)\right]_{i}\right|+\left|p_{i}(t)\right|
$$

Let $\tilde{\xi}_{\text {sum }}(0)=\left|\tilde{\xi}_{1}(0)\right|+\left|\tilde{\xi}_{2}(0)\right|+\left|\tilde{\xi}_{3}(0)\right|$. According to $\varepsilon_{i}(t)=$ $\left(\tilde{\xi}_{i}(t) / \omega_{o}^{i-1}\right)$ and $(18)-(20)$, we have

$$
\left|\tilde{\xi}_{i}(t)\right| \leq\left|\frac{\tilde{\xi}_{\text {sum }}(0)}{\omega_{o}^{3}}\right|+\frac{3 \delta}{\omega_{o}^{4-i}}+\frac{4 \delta}{\omega_{o}^{7-i}}=\sigma_{i}
$$

for all $t \geq T_{1}, i=1,2,3$. Q.E.D.

It is shown that, in the absence of the plant model, the estimation error of ESO (5) is bounded and that its upper bound monotonously decreases with the increase of the observer bandwidth. The assumption of the boundedness of $h(\xi, d)$ means that there is a limit to the rate of change of the total efforts of the internal dynamics and the external disturbances, excluding the control input for the MEMS gyroscope, or that the change is not instantaneous. In this case, $f$ can change very rapidly, and the magnitude of $\dot{f}$ can be quite large although bounded. Understandably, this requires the observer bandwidth to be sufficiently large for an accurate estimate of $f$. In the absence of this boundedness assumption, the rate of change in $f$ would be unlimited, which would make $f$ very difficult to estimate. Fortunately, for MEMS gyroscopes, this assumption seems to be a reasonable one because its mechanical construction and working environment does not allow the acceleration to change instantaneously, thus forcing $\dot{f}$ to be a bounded variable.

The convergence of the ADRC, where ESO is employed, is analyzed next.

2) Convergence of the ADRC: Let $\left[r_{1}, r_{2}, r_{3}\right]^{\mathrm{T}}=[r, \dot{r}, \ddot{r}]^{\mathrm{T}}$ and $e_{i}(t)=r_{i}(t)-\xi_{i}(t), i=1,2$.

Theorem 2: Assuming that $h$ is bounded, there exist a constant $\rho_{i}>0$ and a finite time $T_{3}>0$ such that $\left|e_{i}(t)\right| \leq$ $\rho_{i}, i=1,2, \forall t \geq T_{3}>0, \omega_{o}>0$, and $\omega_{c}>0$. Furthermore, $\rho_{i}=\mathrm{O}\left((1) /\left(\omega_{c}^{q}\right)\right)$ for some positive integer $q$.

Proof: From (7), one has

$$
u_{d}=\frac{k_{1}\left(e_{1}+\tilde{\xi}_{1}\right)+k_{2}\left(e_{2}+\tilde{\xi}_{2}\right)-\left(\xi_{3}-\tilde{\xi}_{3}\right)+r_{3}}{b} .
$$

It follows that

$$
\begin{gathered}
\dot{e}_{1}=\dot{r}_{1}-\dot{\xi}_{1}=r_{2}-\xi_{2}=e_{2} \\
\dot{e}_{2}=-k_{1}\left(e_{1}+\tilde{\xi}_{1}\right)-k_{2}\left(e_{2}+\tilde{\xi}_{2}\right)-\tilde{\xi}_{3} . \\
\text { Let } e(t)=\left[e_{1}(t), e_{2}(t)\right]^{\mathrm{T}} \text { and } \tilde{\xi}(t)=\left[\tilde{\xi}_{1}(t), \tilde{\xi}_{2}(t), \tilde{\xi}_{3}(t)\right]^{\mathrm{T}} \text {, then } \\
\dot{e}(t)=A_{e} e(t)+A_{\tilde{\xi}} \tilde{\xi}(t)
\end{gathered}
$$

where

$$
A_{e}=\left[\begin{array}{cc}
0 & 1 \\
-k_{1} & -k_{2}
\end{array}\right] A_{\tilde{\xi}}=\left[\begin{array}{ccc}
0 & 0 & 0 \\
-k_{1} & -k_{2} & -1
\end{array}\right] .
$$

Solving (24), we have

$$
e(t)=e^{A_{e} t} e(0)+\int_{0}^{t} e^{A_{e}(t-\tau)} A_{\tilde{\xi}} \tilde{\xi}(\tau) \mathrm{d} \tau .
$$

According to (24) and Theorem 1, one has

$$
\begin{aligned}
& {\left[A_{\tilde{\xi}} \tilde{\xi}(\tau)\right]_{i=1}=0} \\
& \left|\left[A_{\tilde{\xi}} \tilde{\xi}(\tau)\right]_{2}\right| \leq k_{\text {sum }} \sigma=\gamma, \quad \text { for all } t \geq T_{1}
\end{aligned}
$$

where $k_{\text {sum }}=1+k_{1}+k_{2}$. Let $\varphi(t)=\int_{0}^{t} e^{A_{e}(t-\tau)} A_{\tilde{\xi}} \tilde{\xi}(\tau) \mathrm{d} \tau$. Define $\Psi=[0 \gamma]^{\mathrm{T}}$. It follows that

$$
\left|\varphi_{i}(t)\right| \leq\left|\left(A_{e}^{-1} \Psi\right)_{i}\right|+\left|\left(A_{e}^{-1} e^{A_{e} t} \Psi\right)_{i}\right|
$$

Since

$$
A_{e}^{-1}=\left[\begin{array}{cc}
-\frac{k_{2}}{k_{1}} & -\frac{1}{k_{1}} \\
1 & 0
\end{array}\right]=\left[\begin{array}{cc}
-\frac{2}{\omega_{c}} & -\frac{1}{\omega_{c}^{2}} \\
1 & 0
\end{array}\right]
$$

we have

$$
\begin{aligned}
& \left|\left(A_{e}^{-1} \Psi\right)_{1}\right|=\frac{\gamma}{\omega_{c}^{2}} \\
& \left|\left(A_{e}^{-1} \Psi\right)_{2}\right|=0 .
\end{aligned}
$$

Since $A_{e}$ is Hurwitz, there exists a finite time $T_{2}>0$ such that

$$
\left|\left[e^{A_{e} t}\right]_{i j}\right| \leq \frac{1}{\omega_{c}^{3}}
$$

for all $t \geq T_{2}, i, j=1,2$. Note that $T_{2}$ depends on $A_{e}$. Let

$$
\begin{aligned}
e^{A_{e} t} & =\left[\begin{array}{cc}
o_{11} & o_{12} \\
& \\
o_{21} & o_{22}
\end{array}\right] \\
e_{\text {sum }}(0) & =\left|e_{1}(0)\right|+\left|e_{2}(0)\right| .
\end{aligned}
$$

It follows that

$$
\left|\left[e^{A_{e} t} e(0)\right]_{i}\right| \leq \frac{e_{\mathrm{sum}}(0)}{\omega_{c}^{3}}
$$

for all $t \geq T_{2}, i=1,2$. Let $T_{3}=\max \left\{T_{1}, T_{2}\right\}$. We have

$$
\left|\left(e^{A_{e} t} \Psi\right)_{i}\right| \leq \frac{\gamma}{\omega_{c}^{3}}
$$


for all $t \geq T_{3}, i=1,2$, and

$$
\left|\left(A_{e}^{-1} e^{A_{e} t} \Psi\right)_{i}\right| \leq\left\{\begin{array}{l}
\left.\frac{1+2 \omega_{c}}{\omega_{c}^{2}} \frac{\gamma}{\omega_{c}^{3}}\right|_{i=1} \\
\left.\frac{\gamma}{\omega_{c}^{3}}\right|_{i=2}
\end{array}\right.
$$

for all $t \geq T_{3}$. From (27), (28), and (32), we obtain

$$
\left|\varphi_{i}(t)\right| \leq\left\{\begin{array}{l}
\frac{\gamma}{\omega_{c}^{2}}+\left.\frac{1+2 \omega_{c}}{\omega_{c}^{2}} \frac{\gamma}{\omega_{c}^{3}}\right|_{i=1} \\
\left.\frac{\gamma}{\omega_{c}^{3}}\right|_{i=2}
\end{array}\right.
$$

for all $t \geq T_{3}$. From (25), one has

$$
\left|e_{i}(t)\right| \leq\left|\left[e^{A_{e} t} e(0)\right]_{i}\right|+\left|\varphi_{i}(t)\right| .
$$

According to (30), (33), and (34), we have

$$
\begin{aligned}
& \left|e_{i}(t)\right| \\
& \leq\left\{\begin{array}{l}
\frac{e_{\text {sum }}(0)}{\omega_{c}^{3}}+\frac{\left(1+2 \omega_{c}+\omega_{c}^{2}\right) \sigma_{i}}{\omega_{c}^{2}}+\left.\frac{\left(1+2 \omega_{c}+\omega_{c}^{2}\right)\left(1+2 \omega_{c}\right) \sigma_{i}}{\omega_{c}^{5}}\right|_{i=1} \\
\left.\frac{e_{\text {sum }}(0)+\left(1+2 \omega_{c}+\omega_{c}^{2}\right) \sigma_{i}}{\omega_{c}^{3}}\right|_{i=2}
\end{array}\right. \\
& \leq \rho_{i}
\end{aligned}
$$

for $t \geq T_{3}, i=1,2$, where $\rho_{i}=\max \left\{\left(\left(e_{\text {sum }}(0)\right) /\left(\omega_{c}^{3}\right)\right)+\right.$ $\left(\left(\left(1+2 \omega_{c}+\omega_{c}^{2}\right) \sigma_{i}\right) / \omega_{c}^{2}\right)+\left(\left(\left(1+2 \omega_{c}+\omega_{c}^{2}\right)(1+\right.\right.$ $\left.\left.\left.\left.2 \omega_{c}\right) \sigma_{i}\right) / \omega_{c}^{5}\right),\left(\left(e_{\text {sum }}(0)+\left(1+2 \omega_{c}+\omega_{c}^{2}\right) \sigma_{i}\right) / \omega_{c}^{3}\right)\right\}$. Q.E.D.

It has been shown that, with plant dynamics being largely unknown, the tracking error and its derivative are bounded, and their upper bounds monotonously decrease with the increase of the observer and controller bandwidths. With the convergence of the tracking error established, we now present the simulation and hardware test results.

\section{Simulation and Hardware Tests}

An FPGA-based digital implementation of the ADRC is designed and conducted on a piezoelectrically driven vibrational beam gyroscope, which is an alternative to the MEMS gyroscope for experimental use. The control algorithm is implemented in custom logic using VHDL.

\section{A. Hardware Setup}

The block diagram of the FPGA-based digital implementation is shown in Fig. 2. The hardware setup includes a core hardware board developed earlier as reconfigurable control and communication module (RCCM) [14], as shown in Fig. 3. It mainly consists of two analog-to-digital converters, each proceeded by an analog programmable filter, a flash memory, and an FPGA chip. The RCCM also supports the Ethernet and controller area network communication. In this implementation, the sinusoidal reference signal, the Nios core processor, and the first-input-first-output buffer are programmed into the FPGA circuit. The control algorithm, ADRC, is also programmed into FPGA using the VHDL language. One external 12-b digital-to-analog converter is employed to convert the digital control signal from FPGA to analog form before it enters the gyroscope circuitry. To close the loop, the output of the drive axis of the gyroscope is first amplified and then fed back to the field-programmable analog array chip on the RCCM. In the

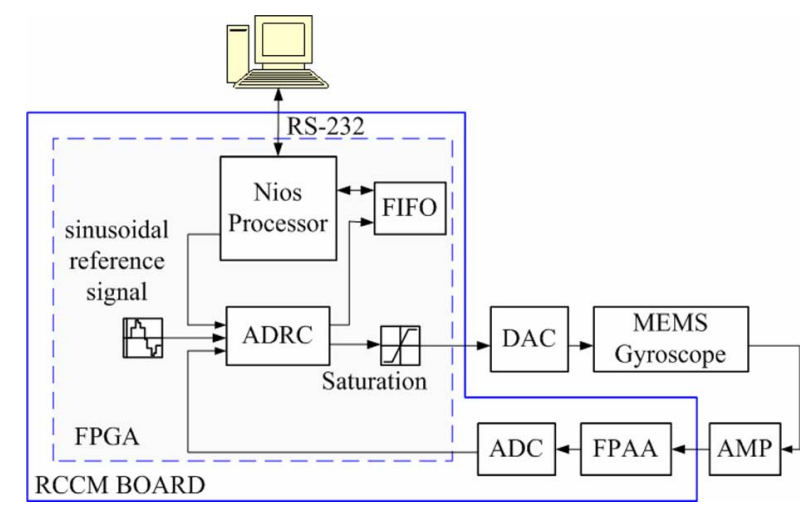

Fig. 2. Control system for the MEMS gyroscope.

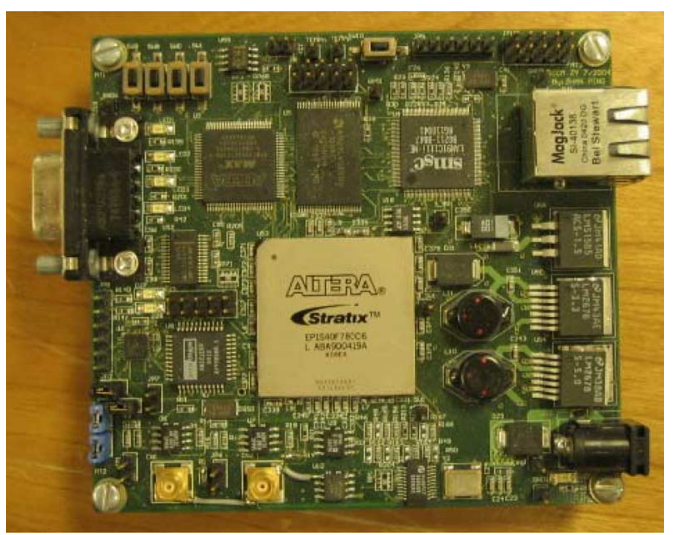

Fig. 3. FPGA-based RCCM.

control system design, the ADRC employs very large controller and observer gains, which are beyond the limited range of integer numbers represented by 32-bit binary. For this reason, the single-precision floating point from IEEE Standard 754 is used for the FPGA-based ADRC design.

In terms of development tools, Quartus II, version 3.2, for FPGA design, SOPC builder for Nios embedded processor design, and GNUPro compiler for building both software and libraries are employed.

\section{B. FPGA Implementation of the ADRC}

For digital implementation, discretizing the state-space model (4) using zero-order hold by ignoring $h$, we have

$$
\left\{\begin{array}{l}
\xi(k+1)=\Phi \xi(k)+\Gamma u_{d}(k) \\
x(k)=H \xi(k)
\end{array}\right.
$$

where

$$
\begin{aligned}
& \Phi=e^{A T_{s}}=\left[\begin{array}{ccc}
1 & T_{s} & \frac{T_{s}^{2}}{2} \\
0 & 1 & T_{s} \\
0 & 0 & 1
\end{array}\right] \\
& \Gamma=\int_{0}^{T_{s}} e^{A \eta} \mathrm{d} \eta B=\left[\begin{array}{c}
\frac{T_{s}^{2} b}{2} \\
T_{s} b \\
0
\end{array}\right] \\
& H=\left[\begin{array}{lll}
1 & 0 & 0
\end{array}\right]
\end{aligned}
$$

and $T_{s}$ is the sampling period.

A discrete ESO is designed as [16]

$$
\hat{\xi}(k)=\bar{\xi}(k)+L_{c}[x(k)-H \bar{\xi}(k)]
$$


where $L_{c}$ is the estimator gain, $\hat{\xi}(k)$ provides a current estimate of $\xi(k)$ based on the current measurement $x(k)$, and $\bar{\xi}(k)$ is the predicted estimate based on a prediction from the previous time estimate, i.e.,

$$
\bar{\xi}(k)=\Phi \hat{\xi}(k-1)+\Gamma u_{d}(k-1) .
$$

Let $\tilde{\xi}(k)=\xi(k)-\hat{\xi}(k)$. It follows that

$$
\tilde{\xi}(k+1)=\left(\Phi-L_{c} H \Phi\right) \tilde{\xi}(k) .
$$

For tuning simplicity, we place all the poles of the desired discrete ESO characteristic equation at $-\beta$, where $\beta=e^{-\omega_{o} T_{s}}$. Then, the desired characteristic polynomial is

$$
\lambda(z)=\left|z I-\Phi+L_{c} H \Phi\right|=(z-\beta)^{3} .
$$

According to Ackermann's formula [16], one has

$$
L_{c}=\left(1-\beta^{3}, \frac{3(\beta-1)^{2}(\beta+1)}{2 T_{s}},-\frac{(\beta-1)^{3}}{T_{s}^{2}}\right)^{\mathrm{T}} .
$$

The discrete implementation of the control law (7) is

$$
u_{d}(k)=\frac{\omega_{c}^{2}\left[r-\hat{\xi}_{1}(k)\right]+2 \omega_{c}\left[\dot{r}-\hat{\xi}_{2}(k)\right]+\ddot{r}-\hat{\xi}_{3}(k)}{b} .
$$

\section{Simulation Results}

The key parameters of the vibrational MEMS gyroscope are $\omega_{n}=63881.1 \mathrm{rad} / \mathrm{s}, \zeta=0.0005$, and $\omega_{x y}=6000 \mathrm{rad}^{2} / \mathrm{s}^{2}$. In (1), $\Omega=\Omega_{0} \sin \left(2 \pi f_{\text {rate }} t\right)$, where $\Omega_{0}$ and $2 \pi f_{\text {rate }}$ are the amplitude and angular frequency of the rate, respectively. The actual amplitude of the rotation rate is assumed to be $0.1 \mathrm{rad} / \mathrm{s}$, and $f_{\text {rate }}$ is assumed to be $50 \mathrm{~Hz}$. The reference signal for the drive axis is $r=A \cos (\omega t)$, where $\omega=63428 \mathrm{rad} / \mathrm{s}$. Typically, the ideal output amplitude of the drive axis is $A=215 \mathrm{mV}$ for the piezoelectrically driven vibrational gyroscope. This voltage output is linearly proportional to the displacement output of the gyroscope in micrometers. We use $A=215$ in "simulation units" to represent the magnitude of the drive axis output in the simulation. The amplitude of the control signal is limited to $\pm 250 \mathrm{mV}$. In the simulation, the mechanical-thermal noise is applied, and the PSD of mechanical-thermal noise is $4.22 \times 10^{-2} \mathrm{~N}^{2} \cdot \mathrm{s}$. The design parameter is $b=k / \mathrm{m}=$ $2.7178 \times 10^{8}$. The controller bandwidth is $\omega_{c}=5 \times 10^{5} \mathrm{rad} / \mathrm{s}$, and the observer bandwidth is $\omega_{o}=2.5 \times 10^{6} \mathrm{rad} / \mathrm{s}$. The sampling period is $T_{s}=1 \times 10^{-8} \mathrm{~s}$.

The output of the drive axis under the control of the discrete ADRC is shown in Fig. 4. After approximately $2.2 \mathrm{~ms}$, the frequency of the drive axis is driven to the resonant frequency $\omega$, as expected. The tracking error between the reference signal $r$ and the output of the drive axis is shown in Fig. 5. The steady-state peak error between the reference and the drive axis output is around $0.17 \%$ of the desired amplitude. Figs. 4 and 5 show the excellent tracking performance of the ADRC.

To further investigate the robustness of the ADRC against parameter variations, the system parameters are changed as fol-

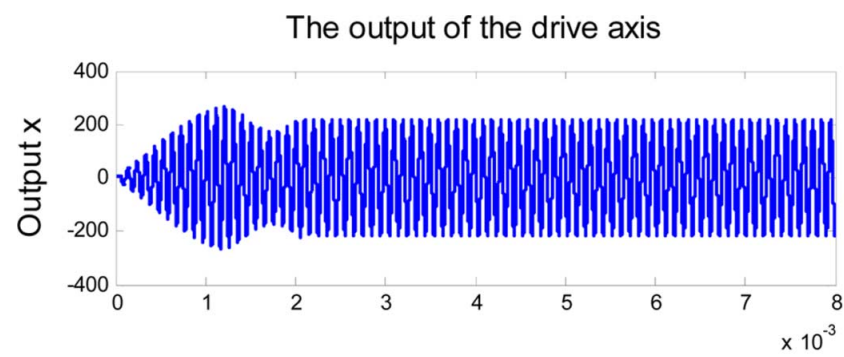

The steady state drive axis output

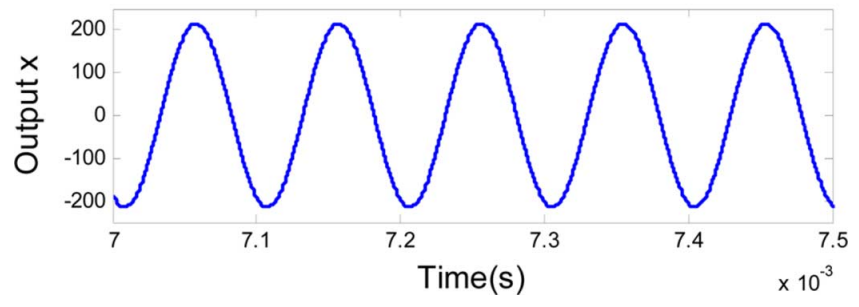

Fig. 4. Output of the drive axis with the ADRC.

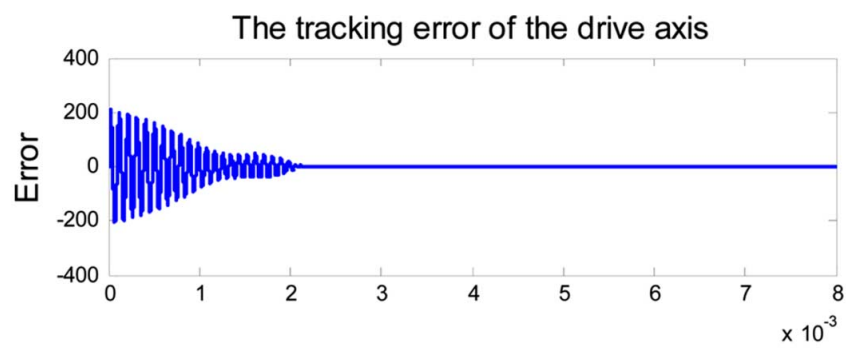

The steady state tracking error of the drive axis

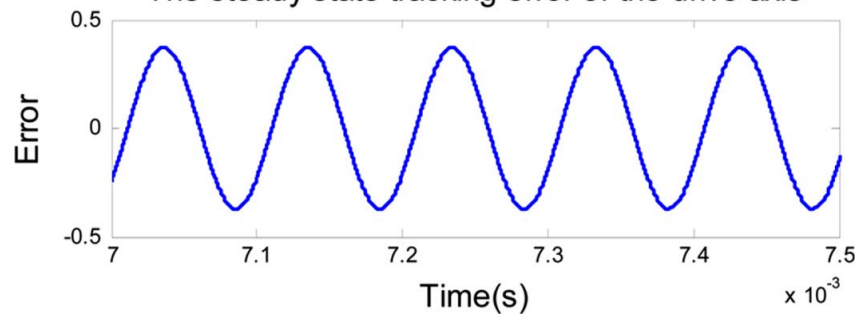

Fig. 5. Tracking error of the drive axis.

lows. The magnitude of the quadrature error term, the damping coefficient, and the frequency of the rotation rate are increased by ten times, i.e., $\omega_{x y}=60000 \mathrm{rad}^{2} / \mathrm{s}^{2}, \zeta=0.005$, and $f_{\text {rate }}=500 \mathrm{~Hz}$, respectively. With these plant parameter variations, the tracking error of the drive axis is shown in Fig. 6. Note that the tuning parameters in the ADRC are the same as that used in Figs. 4 and 5. It can be seen that the frequency of the drive axis is driven to the resonant frequency $\omega$ after approximately $1.8 \mathrm{~ms}$, and the steady-state peak error between the reference and the drive axis output is around $0.17 \%$ of the desired amplitude. With the large-scale plant parameter variations, the performance of the ADRC is almost the same. This shows the strong robustness of the ADRC against parameter variations.

\section{Hardware Test Results}

If the sampling period $T_{s}$ is chosen the same as the one used in the simulation, it will be too fast to implement into the FPGA 

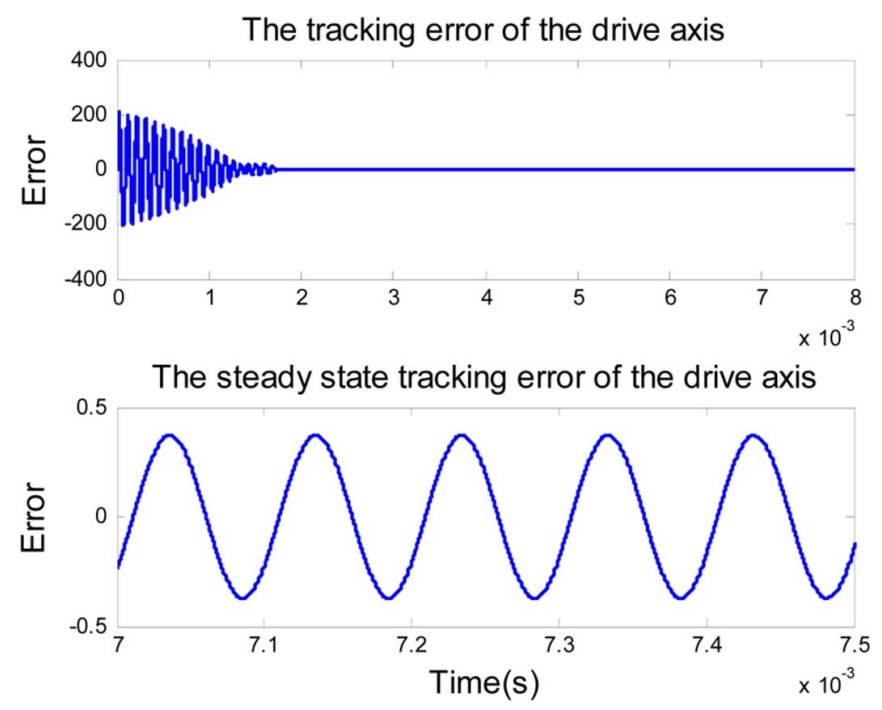

Fig. 6. Tracking error of the drive axis with parameter variations.
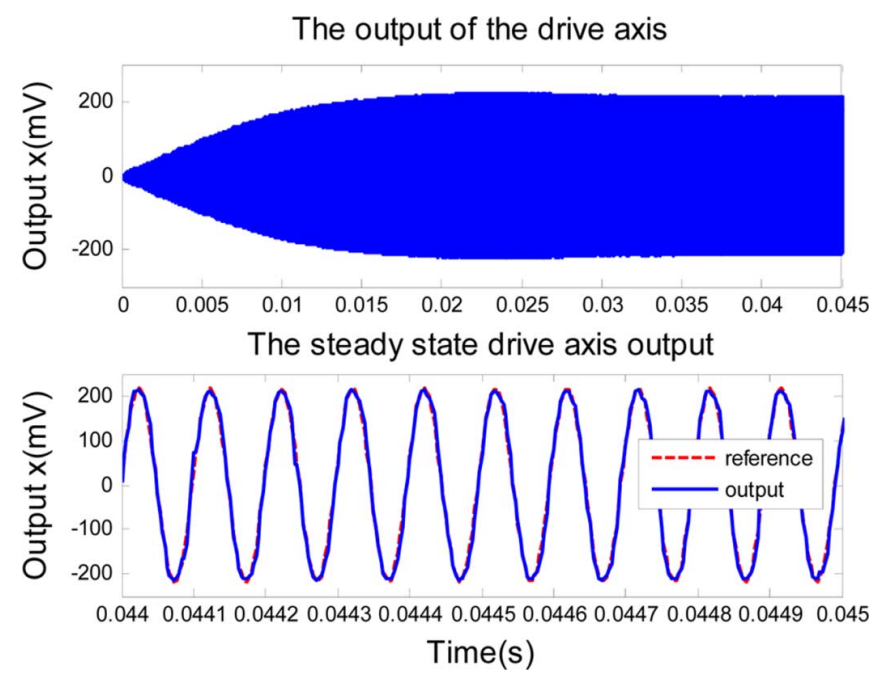

Fig. 7. Drive axis output of the FPGA implementation.

board since the FPGA board clock speed is $50 \mathrm{MHz}$, and the digital ADRC requires several clock cycles to process. For this reason, the tuning parameters are adjusted as the controller bandwidth $\omega_{c}=2.5 \times 10^{6} \mathrm{rad} / \mathrm{s}$, the observer bandwidth $\omega_{o}=2.5 \times 10^{6} \mathrm{rad} / \mathrm{s}$, and the sampling period $T_{s}$ is reduced to $1 \times 10^{-6} \mathrm{~s}$. The output of the drive axis is shown in Fig. 7 . From Fig. 7, it can be seen that the frequency of the drive axis is driven to the resonant frequency $\omega$ after approximately $18 \mathrm{~ms}$. At the steady state, the output matches the reference very well. The steady-state peak error between the reference and the drive axis output is around $0.93 \%$ of the desired amplitude. These show the good performance of the ADRC in the FPGA implementation.

\section{CONCLUSION}

In this paper, a novel concept, namely, active disturbance rejection, is successfully applied to solve the problems in MEMS gyroscopes that stem from manufacturing imperfections. Such imperfections manifest themselves as uncertain dynamics and unknown disturbances that are difficult to deal with using existing design methods that are largely dependent on an accurate mathematical model. The proposed ADRC design proves to be a good fit for three reasons: 1) It requires minimal a priori information of the plant (just the order of the plant and its high frequency gain); 2) it actively estimates and compensates for the unknown dynamics and disturbances; and 3) the controller is easy to be implemented and tuned as compared to other methods. The results obtained from simulation and hardware tests demonstrated the effectiveness of the ADRC. The stability analysis solidified the theoretical foundation of the proposed approach and provided much insight for the users, and the rationale for the success of both simulation and hardware tests.

\section{REFERENCES}

[1] Y. Yazdi, F. Ayazi, and K. Najafi, "Micromachined inertial sensors," Proc. IEEE, vol. 86, no. 8, pp. 1640-1659, Aug. 1998.

[2] R. P. Leland, "Adaptive tuning for vibrational gyroscopes," IEEE Trans. Control Syst. Technol., vol. 11, no. 2, pp. 242-247, Mar. 2003.

[3] R. P. Leland, Y. Lipkin, and A. Highsmith, "Adaptive oscillator control for a vibrational gyroscope," in Proc. Amer. Control Conf., 2003, pp. 3347-3352.

[4] R. T. M'Closkey and A. Vakakis, "Analysis of a microsensor automatic gain control loop," in Proc. Amer. Control Conf., 1999, pp. 3307-3311.

[5] L. Dong and R. P. Leland, "The adaptive control system of a MEMS gyroscope with time-varying rotation rate," in Proc. Amer. Control Conf., 2005, pp. 3592-3597.

[6] R. Leland, "Adaptive control of a MEMS gyroscope using Lyapunov methods," IEEE Trans. Control Syst. Technol., vol. 14, no. 2, pp. 278-283, Mar. 2006.

[7] S. Park, "Adaptive control strategies for MEMS gyroscopes," Ph.D. dissertation, Univ. California, Berkeley, CA, 2000.

[8] S. Park and R. Horowitz, "Adaptive control for the conventional mode of operation of MEMS gyroscopes," J. Microelectromech. Syst., vol. 12, no. 1, pp. 101-108, Feb. 2003.

[9] Z. Gao, "Scaling and parameterization based controller tuning," in Proc. Amer. Control Conf., 2003, pp. 4989-4996.

[10] Z. Gao, "Active disturbance rejection control: A paradigm shift in feedback control system design," in Proc. Amer. Control Conf., 2006, pp. 2399-2405.

[11] Y. X. Su, B. Y. Duan, C. H. Zheng, Y. F. Zhang, G. D. Chen, and J. W. Mi, "Disturbance-rejection high-precision motion control of a stewart platform," IEEE Trans. Control Syst. Technol., vol. 12, no. 3, pp. 364-374, May 2004.

[12] B. Sun and Z. Gao, "A DSP-based active disturbance rejection control design for a $1 \mathrm{KW}$ H-bridge DC-DC power converter," IEEE Trans. Ind. Electron., vol. 52, no. 5, pp. 1271-1277, Oct. 2005.

[13] Q. Zheng and Z. Gao, "Motion control optimization: Problem and solutions," Int. J. Intell. Control Syst., vol. 10, no. 4, pp. 269-276, 2006.

[14] Z. Ping and Z. Gao, "A FPGA-based digital control and communication module for space power management and distribution systems," in Proc. Amer. Control Conf., 2006, pp. 4941-4945.

[15] R. P. Leland, "Mechanical thermal noise in MEMS gyroscopes," IEEE Sensors J., vol. 5, no. 3, pp. 493-450, Jun. 2005.

[16] G. F. Franklin, J. D. Powell, and M. Workman, Digital Control of Dynamic Systems, 3rd ed. Reading, MA: Addison-Wesley, 1997. 